\title{
Bilobectomy for non-small cell lung cancer: A search for clinical factors that may affect perioperative morbidity and long-term survival
}

\author{
Anthony W. Kim, MD, L. Penfield Faber, MD, William H. Warren, MD, Neha D. Shah, MD,
} Sanjib Basu, PhD, and Michael J. Liptay, MD

Objective: The resection of two lobes for non-small cell lung cancer has the potential for significant morbidity and mortality as well as a negative impact on survival. The purpose of this study is to analyze our bilobectomy experience.

\begin{abstract}
Methods: Age, gender, diagnosis, bilobectomy type, bilobectomy indication, operative technique, pathologic condition, major complications, stage, and survival were reviewed from 1984 through 2007. Major complications were compared by Fisher's exact testing. Kaplan-Meier survival curves were compared by log-rank and likelihood ratio analysis.
\end{abstract}

\begin{abstract}
Results: Bilobectomies were performed on 92 patients with non-small cell lung cancer. A total of 35 upper-middle and 57 middle-lower bilobectomies were performed. Indications for bilobectomy were bronchial involvement $(n=49)$, extension across the fissure $(n=36)$, or other reasons $(n=7)$. The 5-year survival for all patients was $42 \%$. Significant differences in survival were observed among the different stages (stage I, $65 \%$; stage II, 42\%; stage III, $13 \% ; P<.0001)$. Squamous cell carcinomas had a higher 5 -year survival than adenocarcinomas $(54 \%$ vs $32 \%)$, a difference that approached significance by $\log$-rank test $(P<.079)$ and reached significance by likelihood ratios $(P<.048)$. When bilobectomy was performed for extension across the fissure, survival approached significance for squamous cell carcinomas $(71 \%)$ over adenocarcinomas $(42 \%)$ by log-rank test $(P<.089)$ and was significant by likelihood ratio $(P<.048)$ when comparing survival between adenocarcinoma and squamous cell carcinoma. Multivariate analysis demonstrated that increasing age $(P=.0102)$ and upper\&middle bilobectomy $(P=.0285)$ adversely affected 5 -year survival, whereas early-stage disease $(P=.0245)$ beneficially affected 5-year survival.
\end{abstract}

Conclusion: Bilobectomy can be performed with acceptable morbidity and mortality. Survival relates to disease stage. Optimal survival benefit occurs when the indication for bilobectomy is squamous cell carcinoma extending across the fissure. (J Thorac Cardiovasc Surg 2010;139:606-11)

It is often accepted that the removal of two pulmonary lobes on the right side, otherwise referred to as a bilobectomy, has an acceptable morbidity and mortality profile. On the basis of the extent of resection, it can be assumed that this morbidity and mortality is intermediate between a lobectomy and pneumonectomy. Presumably, the long-term survival is also reflective of the stage of disease that warranted a bilobectomy. It is unclear whether the indication for the bilobectomy both justifies and affects the performance of this operation. The primary objective of this study was to determine the survival associated with bilobectomy for non-

From the Department of Cardiothoracic-Vascular Surgery, Rush University Medical Center, Chicago, Ill.

Disclosures: None.

Received for publication Sept 27, 2008; revisions received April 13, 2009; accepted for publication May 16, 2009; available ahead of print Aug 26, 2009.

Address for reprints: Michael J. Liptay, MD, 1725 W Harrison St, Suite 774, Chicago, IL 60612 (E-mail: Michael_Liptay@rush.edu).

$0022-5223 / \$ 36.00$

Copyright (c) 2010 by The American Association for Thoracic Surgery doi:10.1016/j.jtcvs.2009.05.044 small cell lung cancer (NSCLC). The secondary purpose was to identify certain clinical factors that affected survival and morbidity.

\section{METHODS}

Clinical records of patients who underwent a bilobectomy at a single academic institution between January 1984 and December 2007 were reviewed. Patients were chosen on the basis of (1) having undergone a successful bilobectomy, (2) having a diagnosis of NSCLC, and (3) having undergone a complete resection of their disease. Patients excluded were those who (1) had a diagnosis other than NSCLC (eg, metastases, neuroendocrine carcinoma, benign disease), (2) had a resection of fewer than two anatomic lobes, and (3) had a resection of greater than two anatomic lobes. Institutional review board approval was obtained to perform this retrospective investigation.

The age, gender, type of bilobectomy (upper and middle [UM] vs middle and lower $[\mathrm{ML}]$ ), stump closure technique, histopathologic diagnosis and stage, perioperative mortality, and postoperative complications were recorded. Tumor stages, irrespective of the date of operation, were adjusted to be in accordance with the current sixth edition of the American Joint Commission for Cancer TNM classification scheme. Operative reports were reviewed to identify the indications for bilobectomy. Bilobectomies were divided into two major categories: (1) those performed secondary to extension across the fissure or (2) those performed secondary to bronchus 

Abbreviations and Acronyms
$\mathrm{ADC}=$ adenocarcinoma
$\mathrm{BPF}=$ bronchopleural fistula
ML = middle and lower
NSCLC $=$ non-small cell lung cancer
SQC = squamous carcinoma
$\mathrm{UM}=$ upper and middle

involvement. Identification of extension of tumor across the fissure was relatively straightforward, whereas classification of bronchial involvement was more complex. Tumors classified under this indication included those that had (1) internal extension of the tumor proximally or distally, (2) external involvement of the tumor encroaching on the bronchus, and (3) involvement of the lymph node between the upper lobe and middle lobe bronchus or between the middle lobe and lower lobe bronchus.

\section{Statistical Methods}

Kaplan-Meier 5-year survival curves were generated for the overall NSCLC population. Kaplan-Meier 5-year survival curves were also generated for univariate comparisons between different subsets, including gender, UM versus ML bilobectomies, stages, nodal status, indications for bilobectomy, and histopathologic subtypes. Additional subset analysis was performed to compare survival between histopathologic subtypes while adjusting for stage and indication for bilobectomy. Log-rank and likelihood ratio analysis were used to compare survivals. The log-rank test (log rank), based on the Mantel-Haenszel statistic, is a nonparametric test that compares the observed and expected number of events at each observed event time. The likelihood ratio test $(-2 \log [\mathrm{LR}])$ is based on the parametric exponential model. ${ }^{1}$ In this article, both the log-rank and the likelihood ratio tests were used with the understanding that the latter used a more stringent statistical model for the hazard functions. Fisher's exact testing was used to compare the incidence of major complications. Multivariate analysis of 5year survival was performed using the Cox proportional hazards regression model. The statistical analysis was performed with SAS version 9.1 (SAS Institute, Inc, Cary, NC) software.

\section{RESULTS}

\section{Univariate Analysis}

Bilobectomies were performed on 92 patients having NSCLC (45 adenocarcinomas [ADC], 39 squamous carcinomas [SQC], and 8 others -4 adenosquamous and 4 large cell carcinomas). A total of 89 bronchial stumps were closed by staples versus 3 closed by suture. Bronchial stump coverage or space reduction techniques were not used. There were $35 \mathrm{UM}$ and $57 \mathrm{ML}$ bilobectomies performed. Indications for bilobectomy included intrinsic or extrinsic bronchial involvement of the tumor $(n=49)$, extension across the fissure $(\mathrm{n}=36)$, or other reasons such as vascular involvement or multiple nodules $(\mathrm{n}=7)$ (Table 1). Intrinsic involvement of the bronchi (38/49) constituted the majority of the tumors that required bilobectomy for this indication. Only $22 \%$ (11/ 49) of the bilobectomies were performed for extrinsic or nodal involvement of the bronchi. The mean age of the 92 patients with NSCLC was $65 \pm 11$ years (range, 37-92 years).
TABLE 1. Indication for bilobectomy

\begin{tabular}{lc}
\hline \multicolumn{1}{c}{ Indication } & No. $(\%)$ \\
\hline Intrinsic or extrinsic bronchial involvement & $49(53 \%)$ \\
Extension across the fissure & $36(39 \%)$ \\
Vascular injury & $(4 \%)$ \\
Multiple nodules & $3(3 \%)$ \\
\hline
\end{tabular}

Major complications included pneumonia or retained secretion requiring intervention (21/92), supraventricular tachyarrhythmia (11/92), prolonged air leak (8/92), empyema (3/92), adult respiratory distress syndrome (2/ 92), thromboembolic complications (2/92), stroke (2/92), stump-related bronchopleural fistula (BPF) (1/92), chylothorax (1/92), myocardial infarction (1/92), and recurrent laryngeal nerve injury (1/92) (Table 2). There was one stump-related BPF that developed in an ML lobe stump closed by suture in a patient who had undergone preoperative concurrent neoadjuvant chemoradiation. This BPF was small and treated successfully with the bronchoscopic instillation of fibrin glue. The remainder had no bronchial complications. There were $4(4.3 \%)$ deaths from pneumonia, empyema, adult respiratory distress syndrome, and pulmonary embolus.

The incidence of major complications among UM versus ML bilobectomies was 31\% (11/35) and 51\% (29/57), respectively ( $P=$ not significant). When complications were analyzed among the indication for bilobectomies, the incidence of complications was 55\% (27/49) for those performed for intrinsic or extrinsic bronchial involvement versus $31 \%(11 / 36)$ for those with extension across the fissure. When the indication for bilobectomy was extension across the fissure, there was a statistically significant increase in the incidence of complications when compared with all other indications combined $(P=.011)$.

Stapled closure of the bronchial stumps was performed on 89 patients with NSCLC. The remaining 3 patients underwent a sutured closure of their bronchial stumps. All 3 of these patients, underwent an ML bilobectomy. Sutured closure was performed in these patients owing to concerns regarding the proximal extent of the tumor, which warranted an opening of the bronchus to ensure clear margins before closure. Therefore, a stapled closure was not initially attempted. One of these 3 patients had a BPF and retained secretions requiring bronchoscopic intervention, and another had a persistent air leak requiring prolong chest tube drainage. The remaining patient did not have a complication. No BPFs developed among the remaining 89 patients with stapled closures. Space reduction techniques, such as pleural tenting or pneumoperitoneum, were not routinely used. Furthermore, stump coverage techniques also were not routinely used.

Chemoradiation was performed in the neoadjuvant setting in 11 patients. Of these 11 patients, 7 underwent an operative procedure to secure a tissue diagnosis. Six patients 
TABLE 2. Major complications among the 92 patients

\begin{tabular}{lc}
\hline \multicolumn{1}{c}{ Complication } & No. $(\%)$ \\
\hline Pneumonia or retained secretion & $21(23 \%)^{*}$ \\
Arrhythmia & $11(12 \%)$ \\
Prolonged air leak & $8(9 \%)^{*}$ \\
Empeyma & $3(3 \%)$ \\
Adult respiratory distress syndrome & $2(2 \%)$ \\
Thromboembolus & $2(2 \%)$ \\
Stroke & $2(2 \%)$ \\
Bronchopulmonary fistulas & $1(1 \%)^{*}$ \\
Chylothorax & $1(1 \%)$ \\
Myocardial infarction & $1(1 \%)$ \\
Recurrent laryngeal nerve injury & $1(1 \%)^{*}$ \\
\hline Some patients had
\end{tabular}

Some patients had more than 1 complication. *Includes 1 with a sutured bronchus (1 sutured closure with 2 complications).

underwent a cervical mediastinoscopy, of which 5 were positive for mediastinal lymph node involvement and 1 was negative for disease. One patient had a supraclavicular lymph node that was positive before chemoradiation. Despite a definitive course of treatment with an interval response to therapy, he had a local recurrence and, therefore, underwent bilobectomy. Of the remaining 4 patients, 3 had enlarged lymph nodes diagnosed primarily by the computed tomographic scan criterion of larger than $1 \mathrm{~cm}$ on short axis. In reality, all 3 of these patients had lymphadenopathy that was much greater than this criterion. One last patient underwent chemoradiation owing to chest wall involvement. Three of these 11 patients had a complication (BPF, $\mathrm{n}=1$; prolonged air leak, $\mathrm{n}=1$; and pulmonary secretions requiring intervention, $\mathrm{n}=1$ ). In this subset of patients, stump coverage also was not routinely used.

Overall 5-year survival for the 92 patients with NSCLC was $42 \%$ (Figure 1). No survival differences were observed when comparing gender and type of bilobectomy (UM vs ML). As expected, significant differences in survival by log-rank and likelihood ratios were observed among the different stages (stage I, $65 \%$; stage II, $42 \%$; stage III, $13 \%$; $P<.0001$ ) (Figure 2). Individually, significant TNM stage survival differences were observed only among the different $\mathrm{N}$ groups and not among the different $\mathrm{T}$ groups. Five-year survival was better for $\mathrm{N} 0$ versus $\mathrm{N} 1$ patients $(62 \%$ vs $35 \% ; P<.020)$ and N0 versus $\mathrm{N} 2(62 \%$ vs $8 \% ; P<$ $.001)$ patients. The difference between $\mathrm{N} 1$ and $\mathrm{N} 2$ patients approached significance $(35 \%$ vs $8 \% ; P<.057)$ (Figure 3$)$.

When overall survival was compared for all NSCLCs, no significant difference in survival was observed when comparing extension across the fissure to bronchial involvement. However, when only tumor histopathologic type was evaluated, SQCs had a higher 5-year survival than ADCs (54\% vs $32 \%)$; this difference approached significance by log rank $(P<.079)$ and reached significance by likelihood ratios $(P<.048)$. When tumor histopathologic type was stratified by indication for bilobectomy, neither ADCs nor SQCs

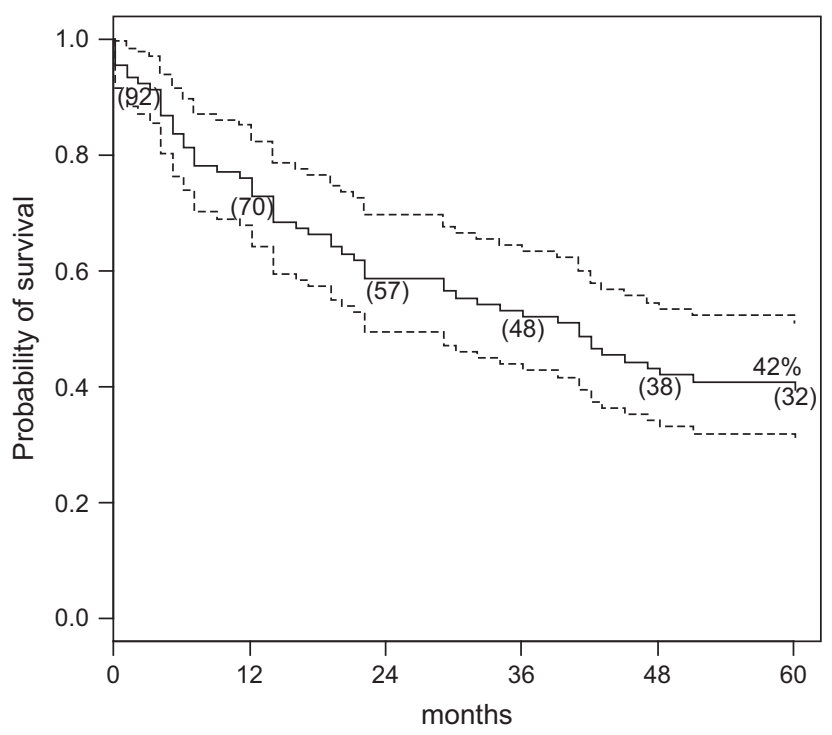

FIGURE 1. Overall 5-year survival of all patients undergoing bilobectomy was $42 \%$. The broken lines represent the $95 \%$ confidence intervals. The numbers in parentheses represent those at risk.

demonstrated a difference in survival when the resection was performed for bronchial involvement or extension across the fissure. The 5-year survival for SQCs, however, when evaluated for extension across the fissure $(71 \%)$ versus for bronchial involvement $(46 \%)$, approached significant difference by likelihood ratio $(P=.067)$. Alternatively, when evaluating the indication for bilobectomy stratified by tumor

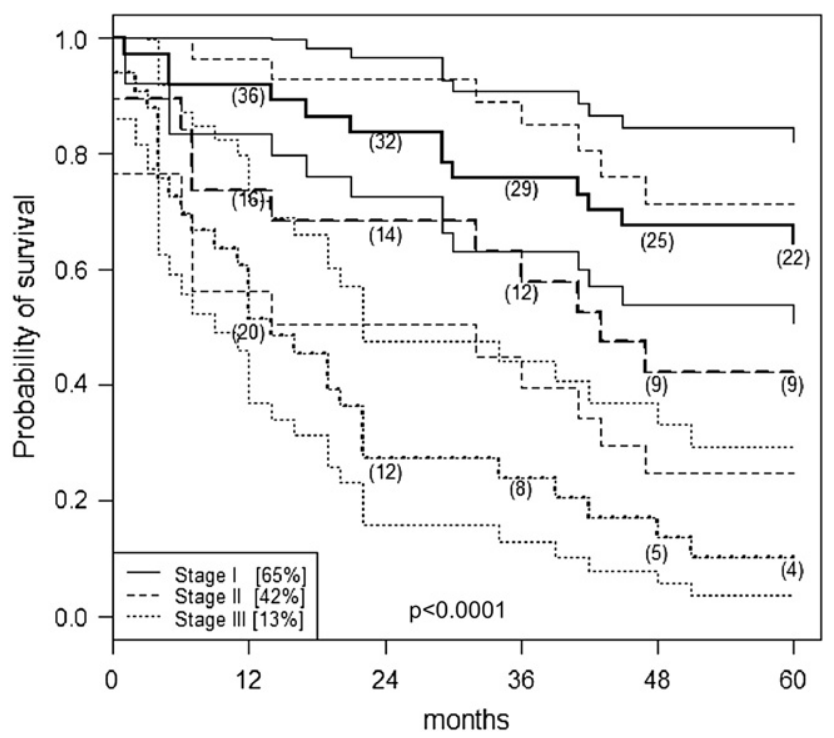

FIGURE 2. Five-year survival stratified by stage of NSCLC. Five-year survival progressively decreases with advancing stage as expected: stage I, $65 \%$; stage II, $42 \%$; stage III, $13 \%$. The additional lines outside the boldface lines represent the $95 \%$ confidence intervals. The numbers in parentheses represent those at risk. 


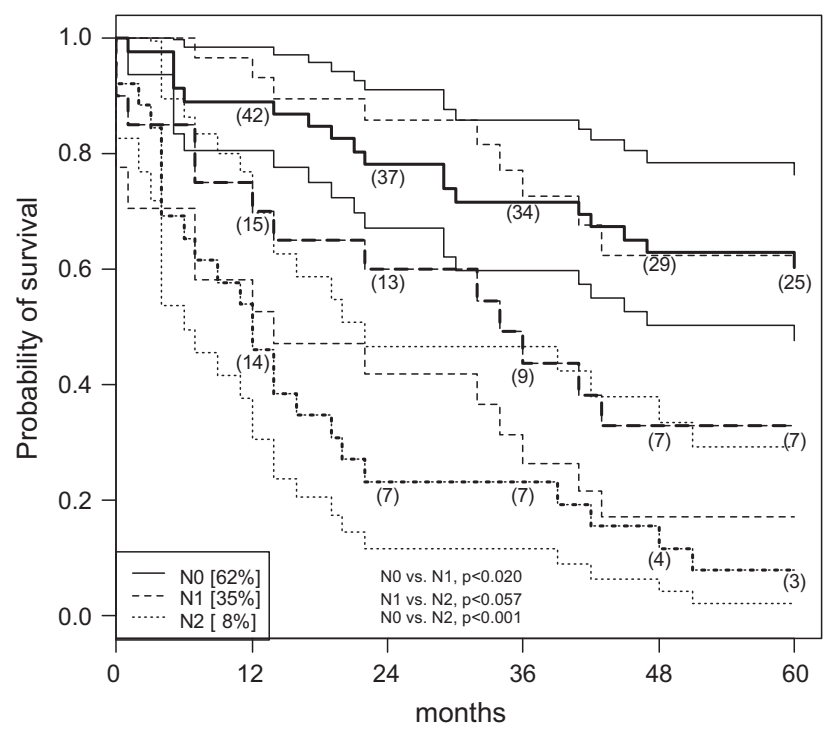

FIGURE 3. Five-year survival stratified by nodal status: (N0 = no nodes, $62 \%$; N1 = ipsilateral hilar nodes, $35 \%$; N2 = ipsilateral mediastinal nodes, $8 \%$. The additional lines outside the boldface lines represent the $95 \%$ confidence intervals. The numbers in parentheses represent those at risk.

histopathologic type, no difference in survival was observed between ADCs and SQCs when bilobectomy was performed for bronchial involvement. However, when bilobectomy was performed for extension across the fissure, the survival difference approached significance for SQCs $(71 \%)$ over ADCs $(42 \%)$ by log-rank analysis $(P<.089)$ and was significant by likelihood ratio $(P<.048)$ (Figure 4$)$.

In another subset analysis, the 5-year survivals for ADCs and SQCs were compared when controlling for both stage and indication. No significant differences in survival between ADCs and SQCs were observed when adjusting for individual stage and indication for bilobectomy. The reason for this most likely was because there were too few numbers for comparison, thereby making for a statistically weak comparison. Even when stage I and II NSCLCs were grouped into early-stage NSCLCs and stage III and IV NSCLCs were grouped into late-stage NSCLCs, a trend toward a difference in survival was not observed between ADCs and SQCs. The lone exception was found within early-stage NSCLCs when bilobectomy was performed for extension across the fissure only. In this subset, a trend toward improved survival for SQCs $(75 \%)$ over ADCs $(46 \%)$ was observed by likelihood ratios $(P=.099)$ (Figure 5).

\section{Multivariate Analysis}

Multivariate analysis demonstrated that increasing age $(P=.0397)$ and UM bilobectomy $(P=.0148)$ adversely affected the 5-year survival. On the other hand and as would be expected, a pathologically negative lymph node status $(P=$ .0032 ) beneficially affected the 5-year survival. Similarly,

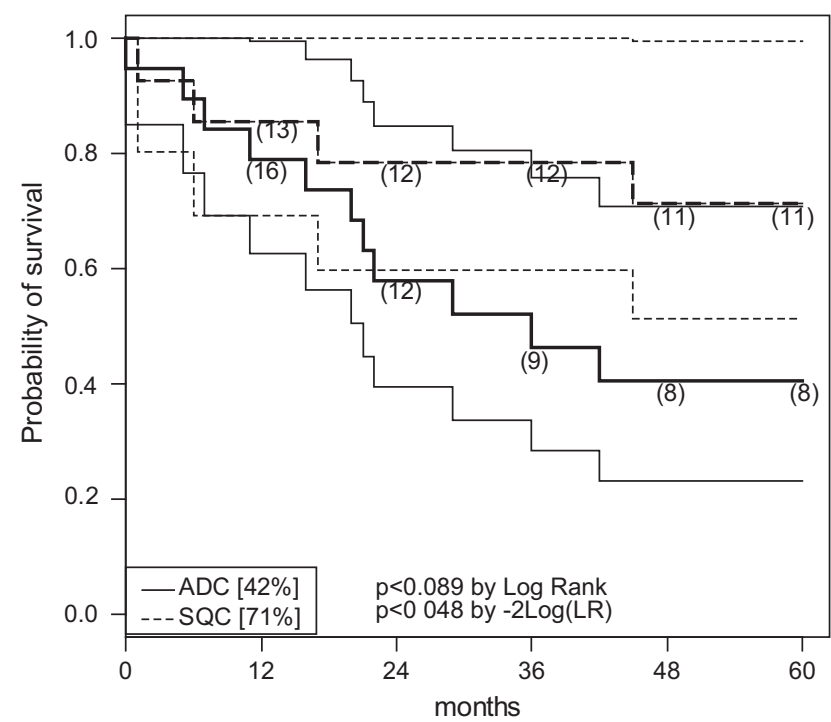

FIGURE 4. Comparison of 5-year survival for adenocarcinomas $(A D C$, $42 \%$ ) versus squamous cell carcinomas (SQC, $71 \%$ ) when the indication for bilobectomy was extension across the fissure, $P=.048$ by likelihood ratio $(-2 \log [\mathrm{LR}])$. The additional lines outside the boldface lines represent the $95 \%$ confidence intervals. The numbers in parentheses represent those at risk.

early stage I disease $(P=.0557)$ approached significance in positively affecting 5 -year survival.

\section{DISCUSSION}

It has been suggested that bilobectomies may be associated with increased morbidity and mortality because of the

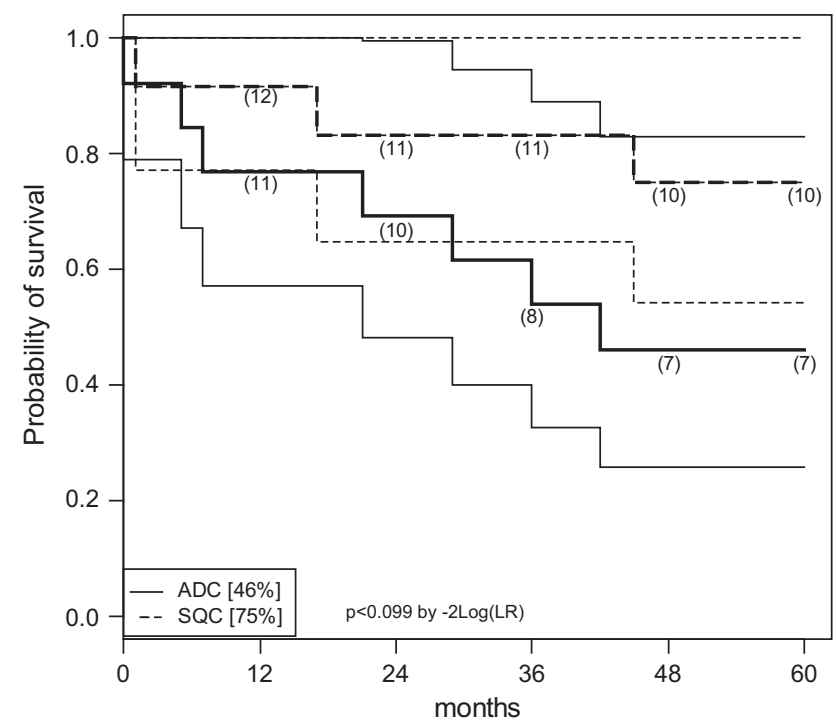

FIGURE 5. Comparison of 5-year survival for early-stage adenocarcinomas $(A D C, 46 \%)$ versus early-stage squamous cell carcinomas ( $S Q C$, $75 \%$ ) when the indication for bilobectomy was extension across the fissure, $P=.099$ by likelihood ratio $(-2 \log \{L R\})$. The additional lines outside the boldface lines represent the $95 \%$ confidence intervals. The numbers in parentheses represent those at risk. 
increased complexity of the resection of an additional lobe rather than resection of a nonanatomic portion of an adjacent lobe. ${ }^{2}$ In fact, the performance of a bilobectomy has been shown to be a risk factor for increased morbidity and mortality in the settings of sleeve resection ${ }^{3}$ and postinduction chemotherapy. ${ }^{4}$ In this study, chemoradiation was not associated with a significantly higher incidence of complications. The overall perioperative mortality risk associated with bilobectomy is reported to be typically between $3.5 \%$ and $6 \%,{ }^{5-8}$ although there have been reports of higher perioperative mortality rates within 60 days. ${ }^{9}$ The overall perioperative mortality of $4.3 \%$ in this series was within the cited range.

In 1991, Vester and associates ${ }^{10}$ reported on a series from our institution of over 2000 pulmonary resections, of which more than $20 \%$ involved a sutured bronchial closure, with an overall incidence of BPFs of $1.6 \%$. Nine BPFs occurred after bilobectomy with sutured closure of the bronchus. In this current series, only 3 patients underwent a sutured closure of the bronchus. Interestingly, a stump-related complication developed in 1 of these $3 \mathrm{pa}-$ tients. The discrepancy noted between this study and the earlier study can be reconciled by the fact that the patients in this series were clinically different from patients analyzed earlier. It could be argued that had the earlier group of patients undergone a stapled closure with the modern day stapling devices, the incidence of BPFs may have been less. Routine coverage of the bronchial stump was not performed in this series. The incidence of BPF in this study was comparable with that reported previously. ${ }^{11-13}$ Interestingly, the BPF that developed in this study occurred in a patient undergoing an ML bilobectomy. This has been cited as a risk factor in their development. ${ }^{13}$

Decreased survival has been associated when bilobectomy is performed for NSCLC. ${ }^{14}$ Bilobectomy appears to have its most negative impact in elderly patients more than 70 years of age. ${ }^{15,16}$ The mean age of the patients in this series approximated the aforementioned age. In this series, tumors crossing the fissure were associated with an improved outcome when compared with those with bronchial involvement. Interestingly, neither the old nor the new staging systems specifically addresses this in their staging schema. Although the issues of visceral pleural involvement and the sequelae of extrinsic or intrinsic lobar involvement are included in the staging system, they do not specifically address tumors that extend across the fissure.

Designating interlobar pleural involvement as T2 or T3 lesions is controversial. ${ }^{2,17-19}$ NSCLCs crossing the fissure into an adjacent lobe have been shown to share a survival profile similar to that of classic T3 tumors. ${ }^{2,17}$ This is in contradistinction to others who have suggested that interlobar pleural involvement warrants a T2 designation, particularly when the lesions can be stratified to SQCs extending across the fissure. ${ }^{18,19}$ The findings of the study reported in this ar- ticle support the contention that SQCs are associated with better long-term survival than other histopathologic subtypes with similar or different indications for bilobectomy.

The extent of surgery, like the T designation, also is a controversial issue. In cases in which the extent of invasion is relatively minimal, ${ }^{2}$ lesser resections of the adjacent involved lobe have been performed. In circumstances with tumor crossing the fissure into the adjacent lobe, lesser resection has still been advocated largely because stage-specific survival is not different between additional lobar versus sublobar resection. ${ }^{17-19}$ Demir and associates ${ }^{2}$ demonstrated that bilobectomy or lobectomy with sublobar resection was associated with significantly better survival when compared with pneumonectomy for adjacent lobar involvement. Unfortunately, the issue of sublobar versus lobar resection remains unanswered inasmuch as there are no randomized controlled data supporting either strategy.

In general, the results of the multivariate analysis were as expected in that increased age negatively affected long-term survival whereas early stage of disease positively affected long-term survival. Although the indication for bilobectomy did not affect 5-year survival, the performance of a UM bilobectomy adversely affected 5-year survival. This finding on multivariate analysis was perplexing and prompted another review of the data (not shown), which did not find a disproportionate or significant number of patients who had advanced age, advanced disease, or any other clinical factor that would indicate this result. Also, the univariate analysis did not mirror these results. This unexpected finding may speak to the other pathophysiologic reasons that may serve as the basis for future studies.

In this article, there were 3 patients with synchronous primary lung cancers that warranted bilobectomy. Deschamps and colleages ${ }^{20}$ showed that bilobectomy for this indication was not only safe, but justified. Bilobectomy for this indication was associated with an increased likelihood of perioperative mortality, but the 5-year survival was similar to that for lobectomy for this indication and statistically different from that for pneumonectomy. ${ }^{15}$ The new staging system downstages these ipsilateral multilobar nodules into the T4 designation, rather than classifying this subset as M1 disease, and also places this group in the stage IIIA rather than the stage IV prognostic category, if there is no N2 or N3 involvement. ${ }^{21}$ This reorganization suggests that resectional therapy plays a role in this subset of patients, and therefore a bilobectomy may be justified. Interestingly, the new staging system does not account for extension across the fissure into an adjacent lobe. This is the very group, when the primary histologic type is SQC, that appears to benefit the greatest from bilobectomy. Further studies for this indication comparing a nonanatomic (wedge) resection of lesser anatomic resection (segmentectomy) versus an anatomic (lobectomy) resection may help to elucidate a potential substage in the new staging system. 
The retrospective nature of this investigation was a major limitation of this study inasmuch as it resulted in the inability to make certain delineations such as that between a larger central mass that crossed the fissure versus one that arose from the two lobar bronchi. However, the medical records, including the rationale for operation and the operative reports, were evaluated carefully and closely to try to best discern between a "bronchial involvement" versus an "extension across the fissure" indication for bilobectomy. Furthermore, in our experience, when a central tumor becomes so large as to make the distinction between bronchial involvement and fissure extension difficult, a more extensive operation, such as a pneumonectomy or sleeve resection, is required, thereby excluding bilobectomy as an option. Similarly, the distinction between extrinsic or nodal involvement was not easily discernible inasmuch as nodal involvement that could not be dissected free on its own was considered equivalent to extrinsic bronchial involvement. In our experience, an extensive nodal dissection is performed throughout any anatomic resection. Therefore, if there is a significant burden of disease that prevents dissection of the lymph nodes separately from the bronchus, then this level of disease is tantamount to extrinsic bronchial involvement. Several subgroup analyses were performed on the basis of the indication for bilobectomy. Qualitatively, the results of these subgroup analyses were similar in that they demonstrated a survival advantage of patients undergoing bilobectomy for SQCs that extended across the fissure. Unfortunately, the differences observed in the subgroup analyses did not reach significance, and this is indicative of the relatively small numbers in subgroups being compared. Last, the statistical analysis performed did not include risk adjustments and, therefore, may have included survival analyses of a heterogeneous population. Despite this, we believed that patient selection was comparable with that which is practiced in other academic institutions and, ultimately, the indication for bilobectomy was the main discerning factor for the performance of this operation, and not other preoperative factors.

Bilobectomies have a morbidity and perioperative mortality profile intermediate between lobectomy and pneumonectomy, but generally approximate historical figures for the former more than the latter. Furthermore, this profile shows that the incidence of complications including a lower incidence of BPF is acceptable when compared historically with pneumonectomy. Ultimately, the findings of this investigation indicate that bilobectomy is a safe operation. The survival suggests that it is an adequate oncologic procedure. Finally, the indication for bilobectomy, particularly if it is an
SQC that extends across the fissure, may be associated with the most benefit when performing bilobectomy.

\section{References}

1. Lawless JF. Statistical methods and methods for lifetime data. New York: John Wiley \& Sons; 1982.

2. Demir A, Gunluoglu MZ, Sansar D, Melek H, Dincer SI. Staging and resection of lung cancer with minimal invasion of the adjacent lobe. Eur J Cardiothorac Surg. 2007;32:855-8.

3. Yildizeli B, Fadel E, Mussot S, Fabre D, Chataigner O, Dartevelle PG. Morbidity, mortality, and long-term survival after sleeve lobectomy for non-small cell lung cancer. Eur J Cardiothorac Surg. 2007;31:95-102.

4. Brouchet L, Bauvin E, Marcheix B, Bigay-Game L, Renaud C, Berjaud J, et al Impact of induction treatment on postoperative complications in the treatment of non-small cell lung cancer. J Thorac Oncol. 2007;2:626-31.

5. Massard G, Dabbagh A, Dumont P, Kessler R, Roeslin N, Wihlm JM, et al. Are bilobectomies acceptable procedures? Ann Thorac Surg. 1995;60:640-5.

6. Keller SM, Kaiser LR, Martini N. Bilobectomy for bronchogenic carcinoma. Ann Thorac Surg. 1988;45:62-5.

7. Deneuville M, Regnard JF, Coggia M, Rojas-Miranda A, Dartevelle P, Levasseur P. The place for bilobectomy in bronchogenic carcinoma. Eur J Car diothorac Surg. 1992;6:446-51.

8. Allen MS, Darling GE, Pechet TT, Mitchell JD, Herndon JE 2nd, Landreneau RJ et al. Morbidity and mortality of major pulmonary resections in patients with early-stage lung cancer: initial results of the randomized, prospective ACOSOG Z0030 trial. Ann Thorac Surg. 2006;81:1013-9; discussion 9-20.

9. Rostad H, Strand TE, Naalsund A, Talleraas O, Norstein J. Lung cancer surgery: the first 60 days. A population-based study. Eur J Cardiothorac Surg. 2006;29:824-8.

10. Vester SR, Faber LP, Kittle CF, Warren WH, Jensik RJ. Bronchopleural fistula after stapled closure of bronchus. Ann Thorac Surg. 1991;52:1253-7; discussion 7-8

11. Sirbu H, Busch T, Aleksic I, Schreiner W, Oster O, Dalichau H. Bronchopleura fistula in the surgery of non-small cell lung cancer: incidence, risk factors, and management. Ann Thorac Cardiovasc Surg. 2001;7:330-6.

12. Sonobe M, Nakagawa M, Ichinose M, Ikegami N, Nagasawa M, Shindo T. Analysis of risk factors in bronchopleural fistula after pulmonary resection for primary lung cancer. Eur J Cardiothorac Surg. 2000;18:519-23.

13. Nagahiro I, Aoe M, Sano Y, Date H, Andou A, Shimizu N. Bronchopleural fistula after lobectomy for lung cancer. Asian Cardiovasc Thorac Ann. 2007;15:45-8.

14. Birim O, Kappetein AP, Bogers AJ. Charlson comorbidity index as a predictor of long-term outcome after surgery for nonsmall cell lung cancer. Eur J Cardiothorac Surg. 2005;28:759-62.

15. van Meerbeeck JP, Damhuis RA, Vos de Wael ML. High postoperative risk after pneumonectomy in elderly patients with right-sided lung cancer. Eur Respir $J$. 2002;19:141-5.

16. Rostad H, Naalsund A, Strand TE, Jacobsen R, Talleraas O, Norstein J. Results of pulmonary resection for lung cancer in Norway, patients older than 70 years. Eur J Cardiothorac Surg. 2005;27:325-8.

17. Okada M, Tsubota N, Yoshimura M, Miyamoto Y, Matsuoka H. How should in terlobar pleural invasion be classified? Prognosis of resected T3 non-small cell lung cancer. Ann Thorac Surg. 1999;68:2049-52.

18. Nonaka M, Kataoka D, Yamamoto S, Horichi N, Ohgiya Y, Kushima M, et al Outcome following surgery for primary lung cancer with interlobar pleural invasion. Surg Today. 2005;35:22-7.

19. Miura H, Taira O, Uchida O, Kato H. Invasion beyond interlobar pleura in nonsmall cell lung cancer. Chest. 1998;114:1301-4.

20. Deschamps C, Pairolero PC, Trastek VF, Payne WS. Multiple primary lung cancers: results of surgical treatment. J Thorac Cardiovasc Surg. 1990;99:769-77; discussion 77-8.

21. Goldstraw P, Crowley J, Chansky K, Giroux DJ, Groome PA, Rami-Porta R, et al The IASLC Lung Cancer Staging Project: proposals for the revision of the TNM stage groupings in the forthcoming (seventh) edition of the TNM Classification of malignant tumours. J Thorac Oncol. 2007;2:706-14. 